\title{
Distribución de Haplaxius crudus (van Duzee, 1907) (Hemiptera: Cixiidae), en las zonas de palma de aceite en Colombia
}

\author{
Distribution of Haplaxius crudus (Van Duzee, 1907) (Hemiptera: Cixiidae), in the oil \\ palm areas of Colombia
}

\author{
(iD NATALIA JULIETH CASTILLO-VILLARRAGA ${ }^{1}$, ID ALEX ENRIQUE BUSTILLO-PARDEY², \\ (iD ANUAR MORALES-RODRÍGUEZ²
}

${ }^{1}$ Cenipalma, Santa Marta, Colombia.ncastillo@cenipalma.org
${ }^{2}$ Cenipalma, Bogotá, Colombia. abustillo@cenipalma.org,amorales@cenipalma.org

*Autor para correspondencia

Natalia Julieth Castillo-Villarraga. Cenipalma, km 64 Vía Santa Marta -Fundación, Troncal de Oriente, municipio de Zona Bananera, Magdalena, Colombia.ncastillo@cenipalma.org

\section{Citación sugerida}

CASTILLO-VILLARRAGA N. J., BUSTILLO-PARDEY A. E., MORALES-RODRÍGUEZ ANUAR. 2022. Distribución de $\mathrm{Ha}$ plaxius crudus (van Duzee, 1907) (Hemiptera: Cixiidae), en las zonas de palma de aceite en Colombia. Revista Colombiana de Entomología 48 (1): e11009. https://doi.org/10.25100/socolen.v48i1.11009

Recibido: 23-Feb-2021

Aceptado: 27-Sep-2021

Publicado: 06-Feb-2022

Revista Colombiana de Entomología ISSN (Print): 0120-0488

ISSN (On Line): 2665-4385

https://revistacolombianaentomologia.univalle.edu.co

Open access

(c) (1) (2) (2) BY-NC-SA 4.0

Publishers: Sociedad Colombiana de Entomología SOCOLEN (Bogotá, D. C., Colombia) https://www.socolen.org.co

Universidad del Valle (Cali, Colombia)

https://www.univalle.edu.co

(C) 2021 Sociedad Colombiana de Entomología SOCOLEN y Universidad del Valle - Univalle
Resumen: La marchitez letal (ML) es una enfermedad de importancia económica de la palma de aceite Elaeis guineensis Jacq., 1897 (Arecales: Arecaceae) en Colombia que ha alcanzado niveles epidémicos en la Zona Oriental palmera, en donde se han registrado 902.529 casos entre 1997 y 2018. El insecto vector de la ML posiblemente es el salta hojas de la palma Haplaxius crudus. El objetivo de este estudio fue obtener información sobre la presencia y distribución de $H$. crudus en las zonas palmeras de Colombia, para lo cual se adelantó una prospección en estas zonas, a través de jameos al follaje de la palma en busca de adultos, y revisando gramíneas y ciperáceas hospederas de las ninfas. Los resultados indican que el insecto está presente en 163 plantaciones de palma de aceite, ubicadas en 42 municipios palmeros distribuidos en las Zonas Palmeras Norte, Central y Oriental de Colombia. Se concluye que la presencia de este insecto es más amplia de lo que se había registrado inicialmente; este trabajo aporta información importante al sector palmero, ya que permite estar alerta sobre la posible diseminación de la ML, una vez que se detecte una palma enferma, en zonas libres de esta enfermedad.

Palabras clave: Colombia, Elaeis guineensis, marchitez letal, palma de aceite, saltahojas, zonas palmeras.

Abstract: Lethal wilt of oil palm (ML, for it name in Spanish) is an economically important disease of the oil palm Elaeis guineensis Jacq., 1897 (Arecales: Arecaceae) in Colombia, where it has reached epidemic levels in the Eastern Palm Zone, with 902,529 cases recorded between 1997 and 2018. The insect vector of ML is probably the cixiid planthopper Haplaxius crudus. The objective of this study was to obtain information on the presence and distribution of $H$. crudus in the oil palm areas of Colombia. Sampling was carried out by passing an insect net to the oil palm foliage, to collect the adults, and checking grasses, and sedge hosts to collect the nymphs. The results indicate that $H$. crudus is present in 163 oil palm plantations, located in 42 palm municipalities distributed in the North, Central, and Eastern oil palm zones of Colombia. We concluded that the presence of this insect is broader than what had been initially recorded. This work provides essential information to the oil palm industry since it allows to be alert about the possible spread of lethal wilt of oil palm once a diseased palm is detected in areas free of this disease.

Key words: Colombia, Elaeis guineensis, lethal wilt disease, oil palm, oil palm zones, planthopper.

\section{Introducción}

Haplaxius crudus (van Duzee, 1907) (Hemiptera: Cixiidae) (= Myndus crudus van Duzee, 1907) es un insecto chupador conocido comúnmente como "salta hojas de la palma" (Bustillo y Arango 2016) y es el principal vector de la enfermedad denominada amarillamiento letal del cocotero (Letal Yellowing LY) (Howard 1983) que ha causado grandes pérdidas en plantaciones de coco (Cocos nucifera L.) en la Florida (Harrison y Elliot 2019), además, podría estar involucrado en la transmisión del virus del decaimiento foliar del coco (CFDV) (Wilson 2005). En Colombia, $H$. crudus reviste importancia, dado que se considera que transmite la enfermedad conocida como Marchitez Letal (ML) (Arango et al. 2011). Esta enfermedad es una de 
las más limitantes en la Zona Oriental y ha alcanzado niveles epidémicos (Bustillo y Arango 2016). Se estima que entre el periodo 2013 y 2018 se han eliminado 3.299 hectáreas afectadas por la Marchitez letal (Tovar 2019).

Este insecto en su estado adulto se alimenta y aparea en el follaje de palmas y sus estados inmaduros se desarrollan en las raíces de gramíneas y ciperáceas. Los adultos de $\mathrm{H}$. crudus han sido encontrados alimentándose en plantas de la familia Arecaceae: C. nucifera, Dypsis lutescens H. Wendl., Phoenix canariensis Hort. Ex Chabaud., Phoenix dactylifera L., Pritchardia pacifica Seem. \& H. Wendl., Pritchardia thurstonii Muell. E. Drude, Pritchardia sp., Roystonea regia Kunth, Sabal palmetto Walter, Trachycarpus fortunei (Hook.) H.Wendl., Veitchia merrillii (Becc.) H.E.Moore., Washingtonia robusta H.Wendl., y Washingtonia sp., mientras las ninfas han sido encontradas en gramíneas (Poaceae Barnhart) y ciperáceas (Cyperaceae Juss.), a saber, Poaceae: Andropogon bicornis L., Andropogon virginicus L., Brachiaria decumbens Stapf., Brachiaria humidicola (Rendle) Schweick., Cenchrus ciliaris L., Chloris barbata Sw., Cynodon dactylon (L.) Pers., Cynodon nlemfuensis Vanderyst., Digitaria eriantha Steud., Digitaria sanguinalis (L.) Scop., Distichlis spicata (L.) Greene., Eleusine indica (L.) Gaertn., Eremochloa ophiuroides (Munro) Hack., Eustachys petraea Sw., Panicum maximum Jacq., Panicum bartowense Scribn. \& Merr., Panicum purpurascens Raddi., Panicum fasciculatum Sw., Paspalum notatum Flugge., Paspalum virgatum L., Setaria viridis (L.) Beauv., Setaria sp. P.Beauv., Stenotaphrum secundatum (Walt.) Kuntze., y Zoysia sp. Willd.; Cyperaceae: Cyperus brevifolius (Rottb.) Hassk., Cyperus ferax (L.) Rich., Cyperus luzulae (L.) Rottb. ex Retz., Cyperus esculentus L. Cyperus sesquiflorus L. y Fimbristylis cymosa Vahl. (CABI 2020; Sierra et al. 2014). En Colombia se han encontrado adultos alimentándose en palma de aceite (E. guineensis) (Arango et al. 2011).

En el continente americano la distribución de $H$. crudus es bastante amplia, se ha registrado desde el sur de Estados Unidos (Florida, Misisipi y Texas), pasando por la Península de Yucatán, Tabasco (Méjico) y en toda América Central (Hill et al. 2018; Narváez et al. 2018; Ramos et al. 2018). En la zona del Caribe se encuentra en Bahamas, Cuba, Haití, Islas Caimán, Jamaica, Puerto Rico, República Dominicana y Trinidad y Tobago (CABI, 2020; Harrison y Elliott 2019). En Suramérica se encuentra en Colombia, Venezuela (Bustillo y Arango 2016) y recientemente ha sido reportada en Brasil (Silva et al. 2019).

En Colombia, se conoce muy poco sobre la distribución de $H$. crudus. Actualmente se cuenta con la información registrada por Bustillo y Arango (2016), en donde el insecto se encontró distribuido en plantaciones ubicadas en el departamento del Meta (municipios: Acacías, Cumaral, San Carlos de Guaroa y San Martín), departamento de Casanare (municipio: Villanueva), departamento de Cundinamarca (municipio: Paratebueno), departamento de Santander (municipio: Puerto Wilches), departamento del Cesar, y una plantación de palma de aceite del departamento del Magdalena. A pesar de su importancia económica, la distribución de $H$. crudus permanece desconocida en muchas de las zonas palmeras del país. El objetivo de esta investigación fue determinar la distribución de H. crudus en las diferentes zonas donde se cultiva la palma de aceite en Colombia.

\section{Materiales y métodos}

Detección de Haplaxius crudus en zonas palmeras. El muestreo para detectar la presencia de $H$. crudus en las plantaciones se realizó en las cuatro zonas palmeras de Colombia (Central, Norte, Oriental y Suroccidental). Los muestreos en cada una de las zonas fueron realizados por asistentes técnicos de Cenipalma previamente capacitados para realizar el muestreo e identificación de los insectos. En 42 municipios se hicieron visitas a 190 plantaciones de palma de aceite (Fig. 1) en las cuales se contaba con permiso de acceso y muestreo. En cada plantación sin importar el área sembrada, se seleccionó un lote de una hectárea en donde se evaluó la presencia o ausencia de adultos en las palmas y de las ninfas en las gramíneas y ciperáceas; los municipios muestreados se detallan en la Tabla 1.

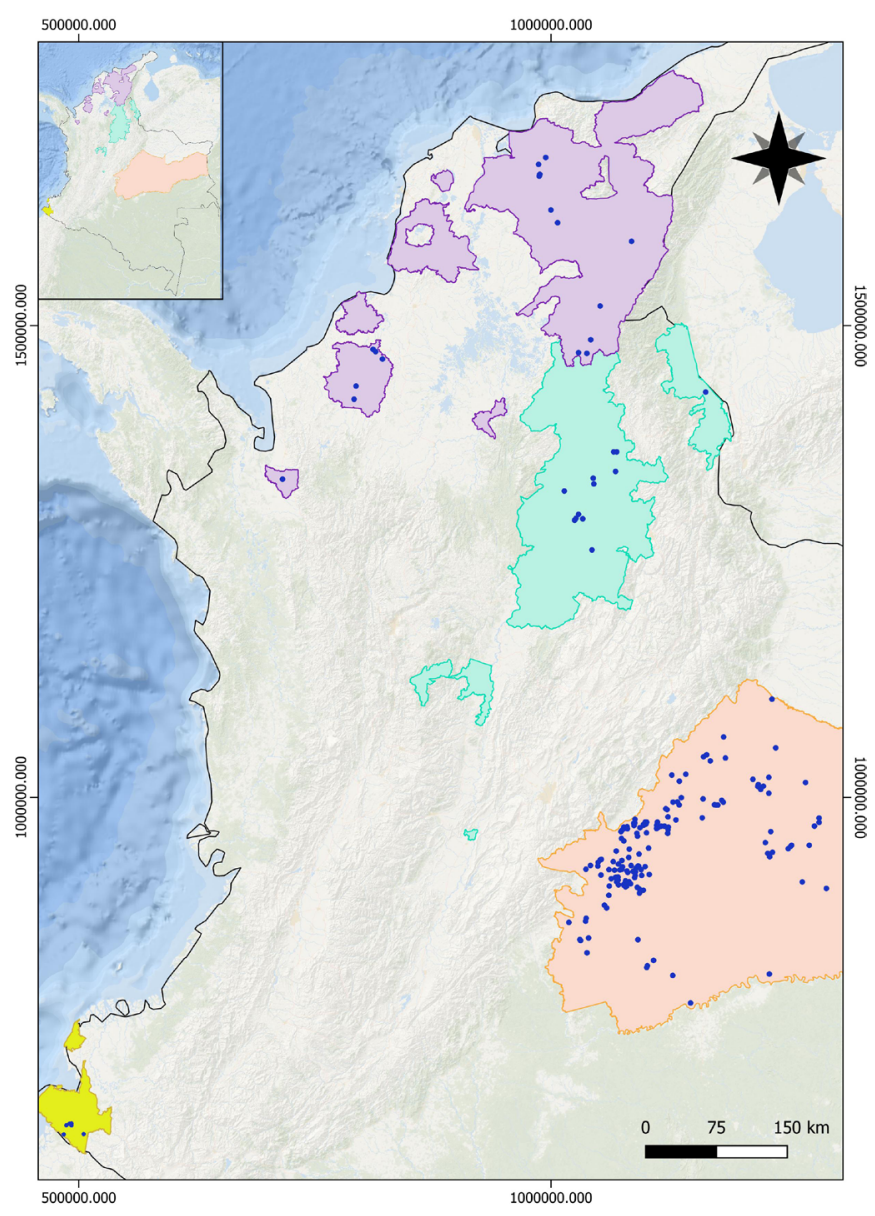

Figura 1. Ubicación de las zonas palmeras y plantaciones en donde se muestreo Haplaxius crudus. Zona Norte: área en morado. Zona Central: área en verde. Zona Oriental: área en naranja. Zona Suroccidental: área en amarillo. Los puntos azules corresponden a los sitios de muestreo.

Se utilizaron diferentes métodos de muestreo para evaluar la presencia del insecto en cada una de las zonas. En las zonas Central, Norte y Suroccidental en donde aún no se ha registrado la presencia de la enfermedad ML, se muestrearon solo adultos utilizando trampas de intersección y jama entomológica dependiendo de la altura de la palma, en la 
zona Oriental en donde está presente la enfermedad se hicieron muestreos tanto de adultos como de ninfas; los adultos se muestrearon usando jama entomológica y trampas de intersección dependiendo de la altura de la palma, mientras que las ninfas se muestrearon revisando raíces de gramíneas y ciperáceas dependiendo de la presencia de estas en el lote (Arango et al. 2012 ).

Muestreo de adultos con jama entomológica. Para evaluar la presencia de adultos de H. crudus, en las plantaciones visitadas se seleccionó una hectárea y se llevó a cabo un muestreo cada cinco líneas y cada cinco palmas (Arango et al. 2012), para un total de 6 palmas por hectárea. Los adultos se colectaron usando una jama entomológica (diámetro del aro: $40 \mathrm{~cm}$, largo: $250 \mathrm{~cm}$ ); en cada palma se hicieron tres pases dobles de jama en el follaje, en los niveles inferior y medio hasta rodear toda la planta (Fig. 2A), equivalentes a dos muestreos por punto cardinal de la planta, para un total de ocho muestreos por palma. Los insectos fueron capturados con un aspirador bucal para su identificación y luego liberados en el campo, en algunas ocasiones se conservaron especímenes que se encuentran depositados en las colecciones.

Muestreo de adultos con trampas de intersección. Se instalaron trampas amarillas para captura de adultos; las trampas se elaboraron siguiendo la metodología propuesta por Arango et al. (2012), con cartón plástico (Cartónplast ${ }^{\circledR}$ ) de $2 \mathrm{~mm}$ de grosor, $25 \mathrm{~cm}$ de alto por $35 \mathrm{~cm}$ de largo y se impregnaron con pegamento agrícola (Pegamosc $\AA$ ) por ambos lados. Las trampas se ubicaron en el borde e interior del lote (dos trampas/ lote), en el borde del plato de la palma a $75 \mathrm{~cm}$ de altura (Fig. 2B) y se revisaron 15 días después.

Muestreo de ninfas. Para evaluar la presencia de ninfas de $H$. crudus, en el borde e interior de lotes se muestrearon macollas de las gamíneas y ciperáceas más abundantes (Bustillo y Arango 2016), a una profundidad de $15 \mathrm{~cm}$ usando una pala jardinera. Luego, se desagregó el suelo y se agitaron

Tabla 1. Municipios muestreados para evaluar la presencia de Haplaxius crudus.

\begin{tabular}{|c|c|c|c|}
\hline Zona palmera & Departamento & Municipios & $\begin{array}{l}\text { Número de } \\
\text { plantaciones } \\
\text { visitadas }\end{array}$ \\
\hline \multirow{5}{*}{ Central } & Bolívar & San Pablo. & 1 \\
\hline & Cesar & San Alberto, San Martín. & 3 \\
\hline & Norte de Santander & Tibú & 1 \\
\hline & Santander & $\begin{array}{c}\text { Barrancabermeja, Rionegro, Sabana de } \\
\text { Torres, y Puerto Wilches. }\end{array}$ & 7 \\
\hline & Antioquia & Chigorodó & 1 \\
\hline \multirow{3}{*}{ Norte } & Cesar & $\begin{array}{l}\text { Agustín Codazzi, El Copey, } \\
\text { Chiriguaná, y Tamalameque }\end{array}$ & 6 \\
\hline & Córdoba & Cereté, Montería, y San Carlos. & 5 \\
\hline & Magdalena & Algarrobo, Aracataca, y Zona Bananera & 5 \\
\hline \multirow[b]{3}{*}{ Oriental } & Casanare & $\begin{array}{l}\text { Aguazul, Maní, Monterrey, Nunchía, } \\
\text { Orocué, Tauramena, y Villanueva. }\end{array}$ & 30 \\
\hline & Cundinamarca & Paratebueno. & 2 \\
\hline & Meta & $\begin{array}{l}\text { Acacías, Barranca de Upía, Cabuyaro, } \\
\text { Castilla La Nueva, Cumaral, Granada, } \\
\text { Puerto Gaitán, Puerto López, Puerto } \\
\text { Lleras, Puerto Rico, San Carlos de } \\
\text { Guaroa, San Juan de Arama, San Mar- } \\
\text { tín, Villavicencio, y Vista Hermosa. }\end{array}$ & 123 \\
\hline Suroccidental & Nariño & San Andrés de Tumaco & 6 \\
\hline
\end{tabular}

las raíces de las plantas sobre un plástico negro (Fig. 2C), para facilitar la visualización de las ninfas (Fig. 2D). Se evaluaron 20 macollas por cada especie de gramínea y ciperácea identificada en el lote.

Identificación del insecto. La identificación inicial de $\mathrm{Ha}$ plaxius crudus se realizó en laboratorio utilizando un estereoscopio Olympus SZ-61 y claves dicotómicas para la familia Cixiidae publicada por Triplehorn y Johnson (2015) y para la especie publicada por Kramer (1979). Algunas caracterís- ticas principales de $H$. crudus son: tamaño no mayor a $5 \mathrm{~mm}$, cuerpo de color pajizo, abdomen de color verde claro, alas hialinas, alas anteriores que no se cubren en reposo (dispuestas en techo), protórax dividido longitudinalmente en cuatro secciones y ojos de color pajizo o negro (dependiendo de la luz). Las ninfas son fácilmente identificables por las siguientes características: color blanco cremoso en sus primeros instares y abdomen de color verde en su último instar, filamentos blancos algodonosos adheridos al dorso de los últimos segmentos abdominales, ojos de color rojo (Zenner De Polania 
y López Ávila 1977; Wilson y Tsai 1982). Los asistentes técnicos de Cenipalma se capacitaron para realizar la identificación del insecto en campo teniendo en cuenta estas características, para lo cual usaron aspiradores bucales y lupas de mano.
Identificación de plantas hospederas. Se recolectaron dos ejemplares por cada especie de planta en donde se encontraron ninfas de $\mathrm{H}$. crudus, los ejemplares se colectaron incluyendo raíces e inflorescencias, estas muestras se prensaron y alcoho-

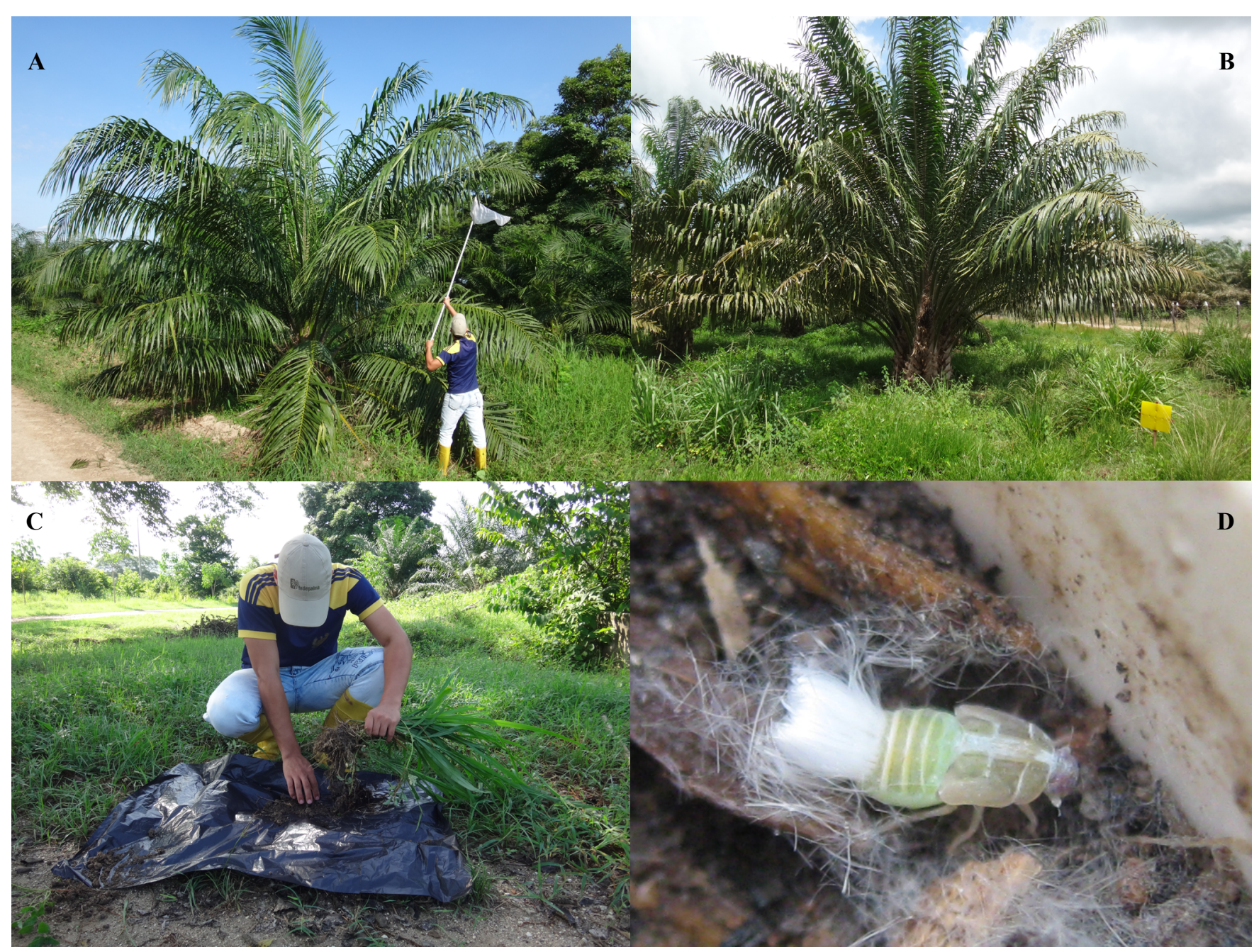

Figura 2. Métodos de muestreo de Haplaxius crudus. A. Pases con jama entomológica en el follaje de la palma de aceite. B. Trampa amarilla en el borde del plato de la palma. C. Revisión de raíces de una macolla de gramínea sobre plástico negro. D. Ninfa de Haplaxius crudus (Foto 2D: C. Sendoya).

lizaron el mismo día de la recolecta para evitar su deterioro y posteriormente se enviaron al Instituto de Ciencias Naturales Herbario Nacional Colombiano (COL), Universidad Nacional de Colombia, sede Bogotá, D.C., para su determinación.

Repositorio de especímenes Boucher. Los ejemplares de las plantas hospederas de ninfas de $H$. crudus se depositaron en el COL (Tabla 2). Algunos de los especímenes de adultos de H. crudus se depositaron en la Colección de Artrópodos Asociados al Cultivo de la Palma de Aceite de Cenipalma (CAACPA) en cada una de las zonas palmeras en donde se registró el insecto.

Tratamiento de datos. Se registraron las coordenadas geográficas de los sitios visitados con un GPS Garmin ${ }^{\circledR}$ S64 y se realizaron mapas de distribución geográfica utilizando el software QGIS 2.18.25.

\section{Resultados}

Distribución de Haplaxius crudus. De las 190 plantaciones visitadas en las cuatro zonas palmeras se detectaron ninfas y adultos de $H$. crudus asociados al cultivo de palma de aceite en 163 plantaciones (Fig. 3A). La especie se encontró en municipios con reportes de ML y municipios sin registro de la enfermedad. En la zona suroccidental no se encontró H. crudus.

Zona Central. Se detectó la presencia de $H$. crudus en todas las plantaciones visitadas (Fig. 3B), los lotes en donde se ins- 
talaron las trampas estaban sembrados con cultivares híbridos interespecíficos OxG y E. guineensis. Las plantaciones visitadas no tenían reportes de casos de ML.

Zona Norte. Se detectó la presencia de H. crudus en todas las plantaciones visitadas (Fig. 3C). Los lotes donde se instalaron las trampas estaban sembrados con cultivares de $E$. guineensis. Las plantaciones visitadas no tenían reportes de casos de ML.

Zona Oriental. En esta zona se realizó el mayor número de visitas, teniendo en cuenta que es la única zona con reportes de ML. Se detectó la presencia de $H$. crudus en 134 plantaciones en 23 municipios, en 78 de estas plantaciones no se habían registrado casos de $\mathrm{ML}$, las 56 plantaciones restantes registraron casos de ML y estaban distribuidas en 10 municipios
(Fig. 3D). Los lotes en donde se realizaron los registros tenían palmas con edades desde los 4 hasta los 31 años de siembra. La mayor cantidad de $H$. crudus se encontró en lotes en donde predominaban las gramíneas, ya fuese dentro del lote o en los bordes. Las gramíneas que más predominaron dependiendo de la edad de cultivo fueron: $P$. virgatum, Megathyrsus maximus (Jacq.) B.K. Simon \& S.W.L. Jacobs y D. sanguinalis.

Cultivares con presencia de adultos de $\boldsymbol{H}$. crudus. El insecto se encontró en plantaciones de palma de aceite, tanto en cultivares de Elaeis guineensis como en híbridos interespecíficos $(\mathrm{OxG})$, sin embargo, fue más frecuente encontrarlo en cultivares de E. guineensis. No obstante, aún no existe evidencia publicada de la preferencia de este insecto por determinados cultivares de palma de aceite. En Cenipalma se están iniciando los primeros trabajos para determinar si

Tabla 2. Especímenes Boucher depositados en el COL

\begin{tabular}{|c|c|c|c|c|}
\hline Nombre & Familia & No. COL & Colector & Determinó \\
\hline Urochloa sp. & Poaceae & 604155 & $\begin{array}{l}\text { Luis Sierra y Natalia } \\
\text { Castillo }\end{array}$ & D. Giraldo Cañas/2018 \\
\hline $\begin{array}{l}\text { Urochlola } \text { cf. brizantha (Hochst. ex A.Rich.) } \\
\text { R.Webster. }\end{array}$ & Poaceae & 604156 & Estiben Caviedes & D. Giraldo Cañas/2018 \\
\hline Paspalum arundinaceum Poir. & Poaceae & 604157 & Estiben Caviedes & D. Giraldo Cañas/2018 \\
\hline $\begin{array}{l}\text { Megathyrsus maximus (Jacq.) B.K. Simon \& } \\
\text { S.W.L. Jacobs. }\end{array}$ & Poaceae & 604158 & $\begin{array}{l}\text { Luis Sierra y Natalia } \\
\text { Castillo }\end{array}$ & D. Giraldo Cañas/2018 \\
\hline Steinchisma laxa (Sw) Zuloaga. & Poaceae & 604159 & $\begin{array}{l}\text { Luis Sierra y Natalia } \\
\text { Castillo }\end{array}$ & D. Giraldo Cañas/2018 \\
\hline Eleusine indica (L.) Gaertn. & Poaceae & 604160 & $\begin{array}{l}\text { Luis Sierra y Natalia } \\
\text { Castillo }\end{array}$ & D. Giraldo Cañas/2018 \\
\hline Paspalum conjugatum P.J. Bergius. & Poaceae & 604162 & $\begin{array}{l}\text { Luis Sierra y Natalia } \\
\text { Castillo }\end{array}$ & D. Giraldo Cañas/2018 \\
\hline Andropogon bicornis L. & Poaceae & 604164 & $\begin{array}{l}\text { Luis Sierra y Natalia } \\
\text { Castillo }\end{array}$ & D. Giraldo Cañas/2018 \\
\hline Kyllinga pumila Michx. & Cyperaceae & 604549 & $\begin{array}{l}\text { Luis Sierra y Natalia } \\
\text { Castillo }\end{array}$ & C. Parra-O/2018 \\
\hline Cyperus luzulae (L.) Rottb. Ex Retz. & Cyperaceae & 604551 & $\begin{array}{l}\text { Luis Sierra y Natalia } \\
\text { Castillo }\end{array}$ & G. Aymard/2018 \\
\hline Rhynchospora nervosa (Vahl) Boeckeler. & Cyperaceae & 604552 & $\begin{array}{l}\text { Luis Sierra y Natalia } \\
\text { Castillo }\end{array}$ & G. Aymard/2018 \\
\hline Cyperus odoratus L. & Cyperaceae & 604543 & $\begin{array}{l}\text { Luis Sierra y Natalia } \\
\text { Castillo }\end{array}$ & D. Giraldo Cañas/2018 \\
\hline
\end{tabular}

H. crudus muestra alguna preferencia por los cultivares híbridos interespecíficos.

Hospederos de ninfas de $\boldsymbol{H}$. crudus. Las ninfas, se encontraron en la base del tallo y la corona de gramíneas y ciperáceas, las especies de gramíneas fueron: A. bicornis, Axonopus compressus, E. indica, Megathyrsus maximus, Paspalum arundinaceum, Paspalum conjugatum, Steinchisma laxa, Urochloa cf. brizantha y Urochloa sp. Por otro lado, las ciperáceas fueron: Cyperus odoratus, Cyperus luzulae, Kyllinga pumila y Rhynchospora nervosa (Vahl) Boeckeler (Fig. 4), esta última es un nuevo registro de hospedero de la familia Cyperaceae albergando ninfas de $H$. crudus (Tabla 2).

\section{Discusión}

Los resultados de este estudio muestran que la presencia de H. crudus en las zonas palmeras de Colombia, es más amplia que lo registrado en la literatura y que este insecto está presente en tres de las cuatro zonas muestreadas. Esto evidencia que la presencia de $H$. crudus no está limitada a la única zona (Oriental) en donde se tienen registros de la enfermedad ML. 
Estos resultados contrastan con lo registrado por Howard et al. (1983) quienes mencionaron que la distribución de H. cru$d u s$, vector del amarillamiento letal del cocotero (LY) en la Florida (USA), coincidía con la distribución conocida de esta enfermedad y enfermedades similares en las Américas.

La enfermedad LY afecta al menos a 36 especies de palmas, siendo $C$. nucifera la más asociada con esta enfermedad (Harrison y Elliot 2019) y H. crudus es considerado su principal vector. En Brasil, afecta plantaciones de C. nucifera (Bastos et al. 2019), en México afecta a P. pacifica (Dzido et al. 2020), C. nucifera y Thrinax radiata Lodd. ex Schult. \& Schult. (Narváez et al. 2018). En Colombia H. crudus se considera que es el vector de la ML (Arango et al. 2011). El manejo de la enfermedad se realiza a través de censos semanales para la detección de palmas enfermas, erradicación de palmas enfermas, aplicación de insecticidas de síntesis química, control de gramíneas y monitoreo de adultos de $H$. crudus a través de trampas pegajosas amarillas y monitoreo de nin- fas desenraizando macollas y revisando sus raíces (Bustillo y Arango 2016). Como parte del manejo de enfermedades transmitidas por vectores, el monitoreo de estos insectos es parte fundamental, ya que provee información acerca de la abundancia, distribución espacial y temporal del insecto en un área epidémica (Mou et al. 2020). En este sentido Howard (1980), encontró que $H$. crudus era 40 veces más abundante en zonas afectadas por el amarillamiento letal en comparación con zonas libres de la enfermedad, así mismo Mou et al. (2020), informaron que la abundancia de H. crudus fue 4,5 veces mayor en palmas enfermas en comparación con palmas sanas. En Colombia, actualmente se desconoce la abundancia de $H$. crudus en las zonas palmeras, por lo cual este trabajo puede servir como base para estudios posteriores en donde se determine la abundancia de este insecto en la zona donde se encuentra la enfermedad y en las zonas sin ML, como parte de una estrategia para controlar la enfermedad y detener su avance hacia nuevas zonas.

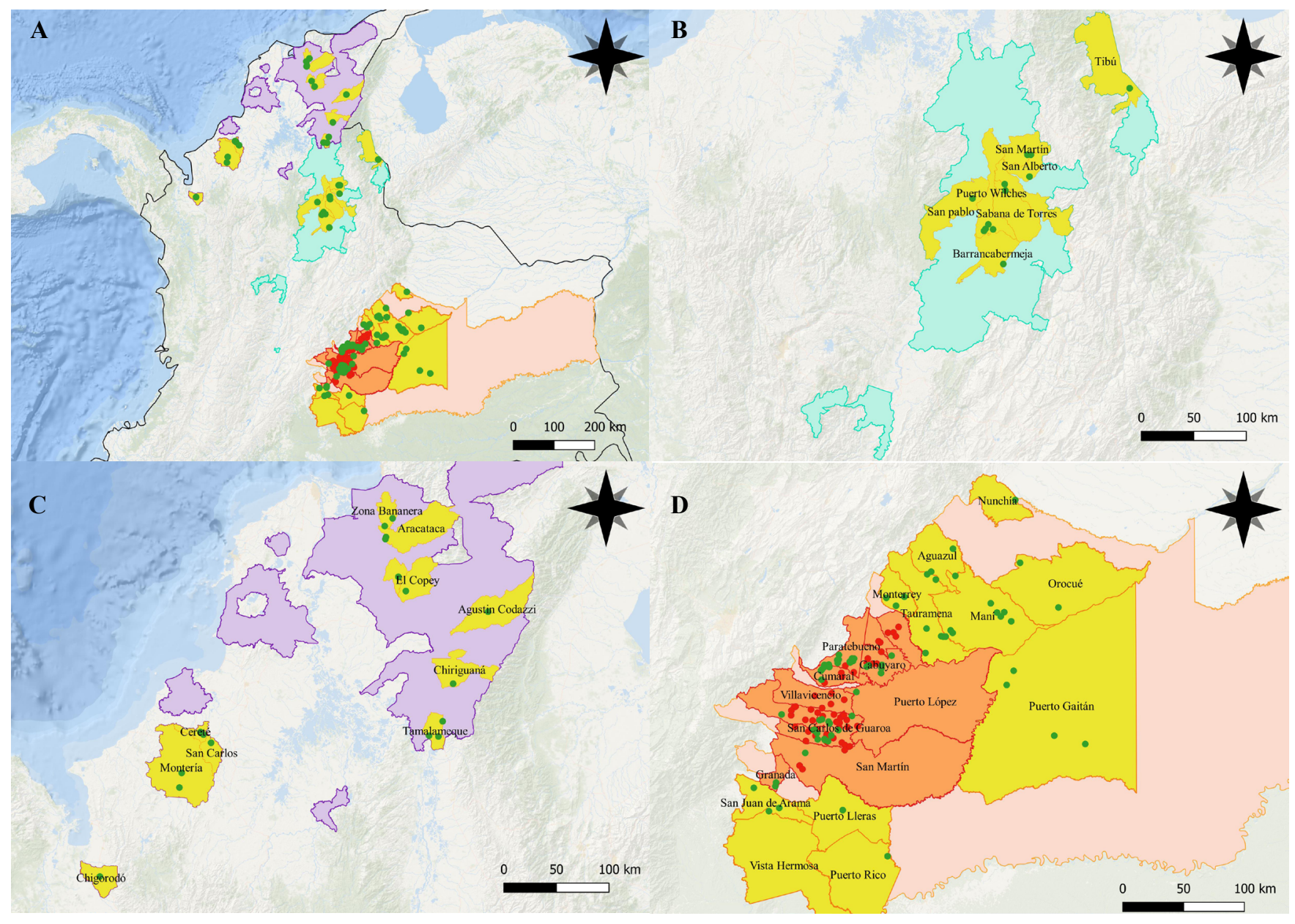

Figura 3. Detalle de la presencia de Haplaxius crudus en las zonas palmeras de Colombia. Municipios de color amarillo: sin registro de ML. Municipios de color naranja: con registro de ML. Puntos de color verde: presencia de H. crudus sin ML. Puntos de color rojo: presencia de H. crudus y ML. A. Presencia H. crudus en Colombia. B. Presencia H. crudus en Zona Central. C. Presencia $H$. crudus en Zona Norte. D. Presencia de H. crudus en Zona Oriental. 


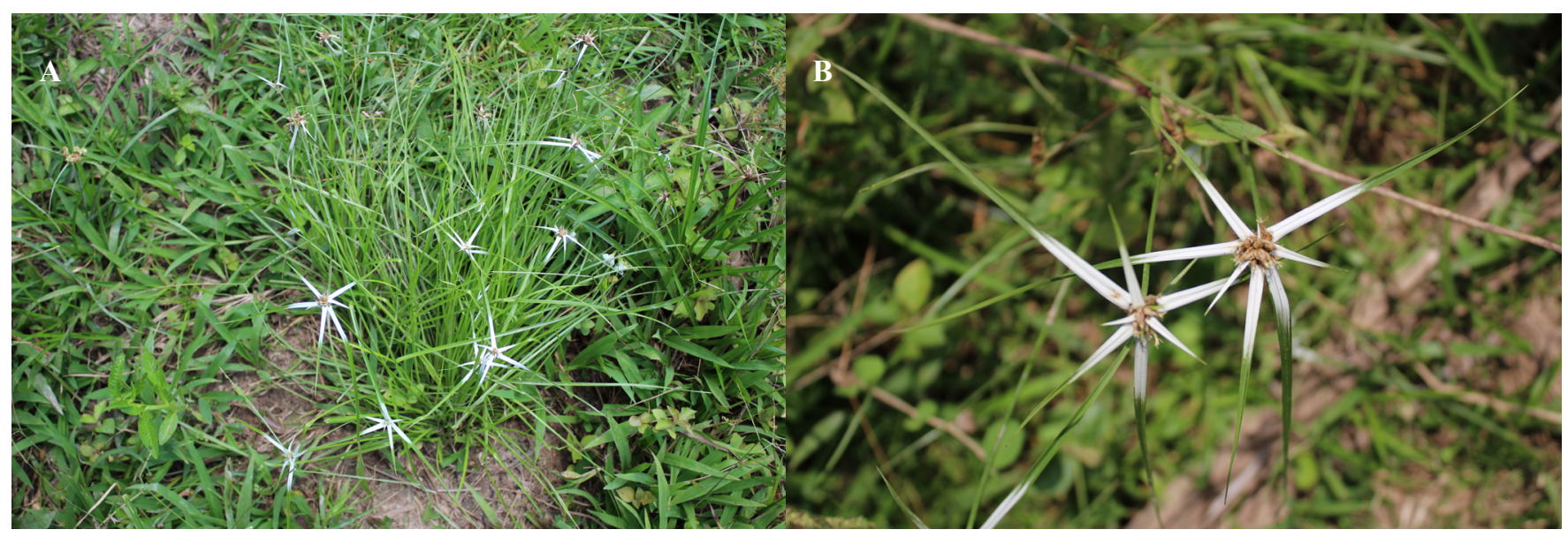

Figura 4. Rhynchospora nervosa. A. Forma de crecimiento. B. Inflorescencia.

\section{Conclusiones}

La información de este estudio relaciona los lugares en que se encuentra la ML y también zonas libres de la enfermedad, en las cuáles $H$. crudus está presente. Esto es muy importante para el palmicultor, ya que les permite estar alerta sobre la posible diseminación de la ML, una vez que se detecte una palma enferma en zonas libres, pero que a la vez se encuentren poblaciones nativas de $H$. crudus.

\section{Agradecimientos}

Los autores agradecen al Fondo de Fomento Palmero, por la financiación de esta investigación. Agradecimientos al personal de las plantaciones visitadas; a los Ingenieros Agrónomos de Cenipalma: Carlos Barrios, Carlos Sendoya, Luis Montes, Luis Sierra y Jesús Matabanchoy, por contribuir a la información sobre la presencia de Haplaxius crudus, en las zonas palmeras y a los revisores anónimos por la revisión del manuscrito.

\section{Literatura citada}

ARANGO, M.; OSPINA, C.; SIERRA, J.; MARTÍNEZ, G. 2011. Myndus crudus: vector del agente causante de la marchitez letal en palma de aceite en Colombia. Palmas (Colombia) 32 (2): 13-25. https://publicaciones.fedepalma.org/index.php/palmas/ article/view/1594/1594

ARANGO, M.; SAAVEDRA, M.; MARTÍNEZ, G. 2012. Efecto del color de las trampas en el monitoreo de adultos de $\mathrm{Ha}$ plaxius (Myndus) crudus. Palmas (Colombia) 33(4): 53-61. https://publicaciones.fedepalma.org/index.php/palmas/article/ view/10740/10727

BASTOS, L.; DOS SANTOS, A.; PENNER, F.; SIQUEIRA, L.; DA SILVA, A.; MARTINS, I.; BATISTA, T. 2019. Spatial analysis and population dynamics of Haplaxius crudus (Hemiptera: Cixiidae) in coconut Amazon. Journal of Agricultural Science 11 (14): 186-197. https://doi.org/10.5539/jas.v11n14p186

BUSTILLO, A.; ARANGO, C. 2016. Las mejores prácticas para detener el avance de la marchitez letal (ML) en plantaciones de palma de aceite en Colombia. Palmas (Colombia) 37 (4): 75-90. https://publicaciones.fedepalma.org/index.php/palmas/article/ view/11965/11957
CABI, 2020. Haplaxius crudus. In Invasive Species Compendium. Wallingford, UK: CAB International. www.cabi.org/isc/datasheetreport/35465. [Fecha de revisión: 20 de diciembre 2020]

DZIDO, J.; SÁNCHEZ, R.; DOLLET, M.; JULIA, J.; NARVAEZ, M.; FABRE, S.; OROPEZA, C. 2020. Haplaxius crudus (Hemiptera: Cixiidae) transmits the lethal yellowing phytoplasmas, 16SrIV, to Pritchardia pacifica Seem. \& H. Wendl (Arecaceae) in Yucatan, Mexico. Neotropical Entomology 49 (6): 795-805. https://doi.org/10.1007/s13744-020-00799-2

HARRISON, N.; ELLIOTT, M. 2019. Lethal yellowing (LY) of Palm. EDIS 2006 (1): 1-8 https://doi.org/10.32473/edis-pp146-2006

HILL, J.; SELTZER, J; HENDON, A.; BARTLETT, C. 2018. First report of the American palm cixiid (Hemiptera: Cixiidae) from Mississippi, USA. Transactions of the American Entomological Society 144(3): 593-597. https://doi.org/10.3157/061.144.0310

HOWARD, F. W. 1980. Population densities of Myndus crudus Van Duzee (Homoptera: Cixiidae) in relation to coconut lethal yellowing distribution in Florida. Principes 24: 174-178. https:// repositorio.fedepalma.org/handle/123456789/80488

HOWARD, F. W.; NORRIS, R.; THOMAS, D. 1983. Evidence of transmission of palm lethal yellowing agent by a planthopper, Myndus crudus (Homoptera, Cixiidae). Tropical Agriculture 60 (3): 168-171. https://www.cabi.org/isc/abstract/19830504423

KRAMER, J. P. 1979. Taxonomic study of the planthopper genus Myndus in the Americas (Homoptera: Fulgoroidea: Cixiidae). Transactions of the American Entomological Society 105 (3): 301-389. https://www.jstor.org/stable/25078242

MOU, D.; LEE, C.; HAHN, P.; SOTO, N.; HUMPHRIES, A.; HELMICK, E.; BAHDER, B. 2020. Effects of lethal bronzing disease, palm height, and temperature on abundance and monitoring of Haplaxius crudus. Insects 11 (11), 748. https://doi. org/10.3390/insects11110748

NARVÁEZ, M.; VÁZQUEZ-EUÁN, R.; HARRISON, N.; NIC-MATOS, G.; JULIA, J.; DZIDO, J.; FABRE, S.; DOLLET, M.; OROPEZA, C. 2018. Presence of 16SrIV phytoplasmas of subgroups A, D and E in planthopper Haplaxius crudus Van Duzee insects in Yucatán, Mexico. 3 Biotech 8 (1): 61. https://doi. org/10.1007/s13205-018-1094-5

RAMOS, E.; MAGAÑA, M.; ORTIZ, C.; OROPEZA, C.; LESHER, J.; SÁNCHEZ, S. 2018. The coconut pathosystem: weed hosts of nymphs of the American palm Cixiid Haplaxius crudus (Hemiptera: Fulgoroidea). Journal of Natural History 52 (5-6): 255-268. https://doi.org/10.1080/00222933.2017.1420832

SIERRA, L.; BUSTILlO, A.; ROSERO, G.; GUTIERREZ, H.; MARTINEZ, J. 2014. Plantas hospederas del vector de la Marchitez letal, Haplaxius crudus, en plantaciones de palma de acei- 
te. Cenipalma. Ceniavances 177: 1-4. https://publicaciones.fedepalma.org/index.php/ceniavances/article/view/11010

SILVA, F.G.; PASSOS, E.M.; DINIZ, L.E.C.; TEODORO, A. V.; TALAMINI, V.; FERNANDES, M.F.; DOLLET, M. 2019. Occurrence in Brazil of Haplaxius crudus (Hemiptera: Cixiidae), vector of Coconut Lethal Yellowing. Neotropical Entomology 48 (1): 171-174. https://doi.org/10.1007/s13744-018-0663-y

TRIPLEHORN, C. A.; N.F JOHNSON. 2005. Borror and DeLong's introduction to the study of insects. Seventh Edition, Thomson Brooks, USA, 864 pp.

TOVAR, J. 2019. Manejo de la enfermedad marchitez letal (ML) bajo el enfoque de principios básicos. Memorias Seminario de actualización técnica sobre el cultivo de palma de aceite. 26 de abril de 2019. Parque vacacional Cofrem Ariari, Granada, Meta. $30 \mathrm{p}$.

WILSON, S. W.; TSAI, J. H. 1982. Descriptions of the immature stages of Myndus crudus (Homoptera: Fulgoroidea: Cixiidae). Journal of the New York Entomological Society 90 (3): 166-175. https://www.jstor.org/stable/25009313

WILSON, S. W. 2005. Keys to the families of Fulgoromorpha with emphasis on planthoppers of potential economic importance in the southeastern United States (Hemiptera: Auchenorrhyncha). Florida Entomologist 88(4): 464-481. https://doi.org/10.1653/ 0015-4040(2005)88[464:KTTFOF]2.0.CO;2

ZENNER DE POLANÍA, I., LÓPEZ AVILA, A. 1977. Apuntes sobre la biología y hábitos del Haplaxius pallidus, transmisor de la marchitez sorpresiva en palma africana. Revista Colombiana de Entomología 3 (1-2): 49-62.

\section{Origen y financiación}

El origen de los datos del presente artículo corresponde a un proyecto de investigación desarrollado por la Corporación Centro de Investigación en Palma de Aceite - Cenipalma y fue financiado por el Fondo de Fomento Palmero.

\section{Contribuciones de los autores}

Todos los autores contribuyeron en mayor o menor medida en la elaboración del presente artículo y sus contribuciones puntuales son las siguientes: Natalia J. Castillo-Villarraga: elaboró protocolo y realizó los experimentos, registro y análisis de datos y escritura del primer manuscrito. Alex E. Bustillo-Pardey: coordino el proyecto, reviso y corrigió el presente manuscrito. Anuar Morales-Rodríguez: asesoró, reviso y corrigió el presente manuscrito. Todos los autores discutieron los resultados y contribuyeron con el manuscrito final.

\section{Conflictos de interés}

Los autores que participan en esta publicación han realizado importantes contribuciones al manuscrito; todos los autores están de acuerdo y expresan que no hay conflictos de intereses en este estudio. 